J. Clin. Chem. Clin. Biochem.

Vol. 26, 1988, pp. 627-633

(C) 1988 Walter de Gruyter \& Co.

Berlin - New York

\title{
Untersuchungen zur Cadmiumbelastung der Bevölkerung im Raum Augsburg (Süddeutschland)
}

\author{
Von H. Gruhl und U. Gilek \\ Institut für Laboratoriumsmedizin, Zentralklinikum Augsburg
}

(Eingegangen am 3. Juni/6. August 1988)

Zusammenfassung: Die Cadmium-Konzentrationen in Leber und Niere wurden bei 68 Verstorbenen (41 männlich, 27 weiblich) aus Süddeutschland (Raum Augsburg) untersucht. Sie lagen in der Leber zwischen 2,2 und 77,3 (Median 16,0) nmol/g Trockengewicht, in der Nierenrinde zwischen 87,2 und 2120,0 (Median 315,4) nmol/g Trockengewicht und im Nierenmark zwischen 34,7 und 913,7 (Median 186,4) nmol/g Trockengewicht. Die Konzentrationen im Nierenmark betrugen im Durchschnitt das 0,56-fache der Nierenrindenwerte. Frauen zeigten in Leber, Nierenrinde und Nierenmark im Median niedrigere Konzentrationen als Männer. Als Gesamtkörperbelastung wurden Werte zwischen 13,2 und 303,9 (Median 51,6) $\mu$ mol ermittelt. Eine mäßige, jedoch signifikante Korrelation wurde für die Cadmium-Konzentrationen in der Leber und der Niere $\left(r_{S}=0,3\right)$, eine stärkere Korrelation zwischen der Nierenmark- und der Nierenrinden-Konzentration $\left(r_{s}=0,6\right)$ gefunden. Auf die Problematik der Umrechnung des Cadmium-Gehaltes in feuchten und trockenen Proben wird eingegangen. Desweiteren wird der Versuch unternommen, die Cadmiumbelastung im Zusammenhang mit einer eventuell vorhandenen Organerkrankung der Leber und/oder der Niere zu sehen.

\section{Investigations on the bodily burden with cadmium in the population of the Augsburg area (Southern Germany)}

Summary: Cadmium concentrations in liver and kidney were determined in 68 deceased patients (41 male, 27 female) from the Augsburg area. The following concentrations were found: liver 2.2-77.3 (median 16.0) $\mathrm{nmol} / \mathrm{g}$ dry weight; renal cortex 87.2-2120.0 (median 315.4) $\mathrm{nmol} / \mathrm{g}$ dry weight; renal medulla 34.7-913.7 (median 186.4) nmol/g dry weight. On average, the values for the renal medullas were 0.56 times the values for the renal cortex. Females showed lower values in liver, renal cortex and renal medulla than males. The total bodily burden with cadmium was determined to be 13.2-303.9 (median 51.6) $\mu$ mol. A moderate, but significant correlation was found between the cadmium concentrations in the liver and the kidney $\left(r_{s}=0.3\right)$, a stronger correlation between the concentrations in the renal cortex and renal medulla $\left(r_{s}=0.6\right)$. The problematic conversion of the results obtained with wet samples into values for dry samples is discussed, as well as the possible influence of different diseases of the liver and/or the kidney on the concentrations found.

\section{Einführung}

Cadmium stellt in der modernen Industrie- und Agrargesellschaft eine Gefährdung für den Menschen und seine Mitwelt dar (Übersichten: 1. c. (1, 2)). Weltweit wurden $1980 \mathrm{ca} .20000 \mathrm{t}$ Cadmium produziert und zu Pigmenten (ca. 30\%), Stabilisatoren für Polyvinylchlorid (PVC) (ca. 18\%), Nickel-Cadmiumbatterien (ca. 15\%), Legierungen (ca. 5\%), und sonstigen
Produkten wie Gleichrichtern, Glas etc. (ca. 12\%) verarbeitet, während in der Galvanotechnik ca. $20 \%$ verbraucht wurden (2). Der Cadmiumeintrag in die Umwelt über Abluft, Abwasser, Abfall und Phosphatdünger wurde für die Bundesrepublik Deutschland für das Jahr 1980 auf ca. $475 \mathrm{t}$ geschätzt (2). Während die akute Toxizität wegen verbesserter arbeitshygienischer Vorkehrungen heute im allgemeinen 
eine untergeordnete Rolle spielt, richtet sich das Interesse vermehrt der chronischen Toxizität des Cadmiums zu. Wird das carcinogene und teratogene Potential des Cadmiums noch kontrovers diskutiert (1, $3)$, so ist die schädigende Wirkung auf Niere $(1,3,5$, $7)$ und Skelettsystem $(1,2)$ heute unumstritten.

Zur Beurteilung einer eventuellen Cadmium-Intoxikation wird üblicherweise die Cadmiumkonzentration im Blut als ein Kurzzeitindikator und die Cadmiumausscheidung im Urin als Marker für eine länger zurückliegende Exposition herangezogen. Für den Raum Augsburg haben wir in einer früheren Arbeit entsprechende Werte für ein Kollektiv gesunder und nierenkranker Personen präsentiert (6). In der vorliegenden Arbeit wird die Cadmiumbelastung der Bevölkerung in dieser Region ermittelt. Hierzu wurden die Cadmiumkonzentrationen in Leber, Nierenrinde und Nierenmark bestimmt und hieraus die Cadmiumbelastung errechnet. Es wird untersucht, inwieweit Korrelationen zwischen den genannten Größen bestehen.

\section{Material und Methoden}

\section{Untersuchungsgut und Probennahme}

Im Rahmen von Sektionen im Pathologischen Institut des Zentralklinikums Augsburg im Jahre 1985 wurden von 41 männlichen Verstorbenen im Alter von 20-82 Jahren und 26 weiblichen Verstorbenen im Alter von 20-84 Jahren Leber- und Nierenproben entnommen. Leber- und Nierengewicht, sowie eventuell pathologische Leber- und Nierenbefunde, wurden den Sektionsprotokollen entnommen. Ungefähr 0,5 Gramm Gewebe von Leber, Nierenrinde und Nierenmark wurden in Glasgefäße überführt. Anschließend wurden die Proben im Gefriertrockner L 05 (Fa. WKF) ca. 12 Stunden lyophilisiert und unter Vakuum verschlossen. Auf kontaminationsfreies Arbeiten wurde geachtet.

\section{Atomabsorptionsspektrometrie (AAS)}

Es wurde am Atomabsorptionsgerät SM 1 der Firma Grün, Wetzlar gearbeitet. Das Gerät verfügt über eine Zeeman-Untergrundkompensation, das Probenschiffchen aus Graphit stellt eine L'vov-Plattform dar. Das AAS Gerät SM 1 verfügt über keine direkte Temperaturmessung am Graphitrohr. Es wird daher auf minimale Verluste bei der Veraschung und maximale Ausbeute bei der Atomisierung experimentell optimiert. Ebenso wird Lampenheizung und -strom sowie der Phasenabgleich am Oszilloskop optimiert. $\mathrm{Da}$ dies für jedes Gerät unterschiedlich ist, verzichten wir auf die Angabe von Geräteparametern.

An das Gerät angeschlossen war eine Waage ME 30 (Firma Mettler, Gießen), auf der die Proben auf dem Graphitschiffchen abgewogen wurden. Die Meßdaten wurden über ein Interface an das AAS-Gerät transferiert. Die lyophilisierten Organproben wurden direkt, ohne weitere Probenvorbereitung, vermessen. Folgende Mengen wurden eingesetzt: Leber 0,2-0,4 mg; Nierenrinde 0,1-0,3 mg; Nierenmark 0,2-0,9 mg.

Die Kalibrierung erfolgte mit wäßrigen Standards, gemessen wurde bei der Wellenlänge $326,1 \mathrm{~nm}$. Als Qualitätskontrolle wurde der NBS Standard Nr. 1577a Rinderleber (Firma Winnopal, Hannover) mitgeführt. Jede Probe wurde dreifach vermessen.
Für die Präzision in der Serie wurde für den NBS Standard $(x=0,44 \mu \mathrm{g} / \mathrm{g})$ ein Variationskoeffizient von $8,4 \%$ gefunden, von Tag zu Tag lag der VK bei $11,2 \%$.

Berechnung der Gesamtkörperbelastung mit Cadmium

; 1

Der Berechnung der Gesamtkörperbelastung liegt die Annahme zugrunde, daß sich ca. die Hälfte des Gesamtkörpercadmiums in der Leber und in den Nieren befindet $(8-10)$. Desweiteren wurde davon ausgegangen, daß die Nierenrinde zu ca. $65 \% \mathrm{am}$ Gewicht der Gesamtniere beteiligt ist (11), und daß die Cadmiumkonzentration in der Nierenrinde ca. das 1,25-fache der Cadmiumkonzentration der Gesamtniere beträgt (11). Es ergibt sich somit folgende Formel für die Körperbelastung mit Cadmium, ausgedrückt in $\mu \mathrm{mol}$ :

$2 \cdot\left(C_{\underline{L}} \cdot f_{L}+C d_{N R} \cdot F_{N} \cdot G_{N} \cdot f_{N}\right) \cdot 0,001$

$\mathrm{Cd}_{\mathrm{L}} \quad=$ Cadmiumkonzentration in der Leber (nmol/g Trokkengewicht)

$\mathrm{Cd}_{\mathrm{NR}}=$ Cadmiumkonzentration in der Nierenrinde (nmol/g Trockengewicht)

$\mathrm{G}_{\mathrm{L}} \quad=$ Gewicht der Leber (g)

$\mathrm{G}_{\mathrm{N}} \quad=$ Gewicht der Nieren (g)

$\mathrm{f}_{\mathrm{L}} \quad=$ Umrechnungsfaktor Lebergewicht feucht-trocken $=0,24$

$\mathrm{f}_{\mathrm{N}} \quad=$ Umrechnungsfaktor Nierengewicht feucht-trocken $=0,21$

$\mathrm{F}_{\mathrm{N}}=$ Umrechnungsfaktor Cadmium-Konzentration in der Nierenrinde auf Cadmium-Konzentration in der Gesamtniere $=0,8$

Ermittlung des Umrechnungsfaktors von Feuchtgewicht auf Trockengewicht

Von vier Verstorbenen (63 Jahre männlich, 26 Jahre männlich, 80 Jahre männlich, 66 Jahre weiblich) wurden jeweils 3 Proben von Leber, Nierenrinde und Nierenmark entnommen. Das Feuchtgewicht der Proben lag bei 1-5 g. Alle Proben wurden sofort nach Entnahme gewogen und anschließend 24 Stunden lyophilisiert. Nach der Gefriertrocknung wurden die Proben erneut gewogen.

Durch Division des Trockengewichtes durch das Feuchtgewicht wurde der Umrechnungsfaktor bestimmt. Als gerundete Mittelwerte für den Umrechnungsfaktor Feucht-Trockengewicht ergaben sich für:

$\begin{array}{ll}\text { Leber } & \mathrm{f}_{\mathrm{L}}=0,24 \\ \text { Nierenrinde } & \mathrm{f}_{\mathrm{NR}}=0,20 \\ \text { Nierenmark } & \mathrm{f}_{\mathrm{NM}}=0,22\end{array}$

\section{Ergebnisse}

Cadmium in der Leber, in der Nierenrinde, im Nierenmark sowie Gesamtkörperbelastung

Die Häufigkeitsverteilungen in der Leber, in der Nierenrinde und im Nierenmark folgen einem log-normalen Modus.

Da viele der Verstorbenen an einer Leber- und/oder Nierenerkrankung litten, werden die Werte nicht zur Ermittlung von Referenzwerten herangezogen. Jedoch errlauben die gemessenen Werte die Beurteilung des durchschnittlichen Cadmiumgehältes der Leber und 
Nieren und schließlich die Berechnung der Gesamtkörperbelastung an Cadmium in der älteren Bevölkerung der Region Augsburg.

Die Ergebnisse der Bestimmung der Cadmiumkonzentration in der Leber, Nierenrinde und im Nierenmark, sowie der daraus berechneten Gesamtkörperbelastung sind in den Tabellen 1-4 zusammengefaßt.

Tab. 1. Cadmium-Konzentration in der Leber (nmol/g Trokkengewicht)

\begin{tabular}{llllll}
\hline & $\begin{array}{l}\text { Alter } \\
\overline{\mathbf{x}}(\mathrm{a})\end{array}$ & Median $\overline{\mathrm{x}}$ & $\mathrm{s}$ & Bereich \\
\hline $\begin{array}{l}\text { Alle Proben } \\
\mathrm{n}=68\end{array}$ & 63,7 & 16,0 & 20,6 & 15,3 & $2,2-77,3$ \\
$\begin{array}{l}\text { Proben }{ }^{2} \\
\mathrm{n}=41\end{array}$ & 61,8 & 16,9 & 24,2 & 17,6 & $2,2-77,3$ \\
$\begin{array}{l}\text { Proben } 9 \\
\mathrm{n}=27\end{array}$ & 64,6 & 11,8 & 15,1 & 8,6 & $5,4-33,5$ \\
\hline
\end{tabular}

Tab. 2. Cadmium-Konzentration in der Nierenrinde (nmol/g Trockengewicht)

\begin{tabular}{llllll}
\hline & $\begin{array}{l}\text { Alter } \\
\overline{\mathbf{x}}(\mathrm{a})\end{array}$ & Median $\overline{\mathrm{x}}$ & $\mathrm{s}$ & Bereich \\
\hline $\begin{array}{l}\text { Alle Proben } \\
\mathbf{n}=68\end{array}$ & 63,7 & 315,4 & 419,9 & 320,7 & $87,2-2120,1$ \\
$\begin{array}{l}\text { Proben } \delta \\
\mathrm{n}=41\end{array}$ & 61,8 & 450,2 & 507,2 & 372,1 & $100,5-2120,1$ \\
$\begin{array}{l}\text { Proben } 9 \\
\mathrm{n}=27\end{array}$ & 64,6 & 261,6 & 287,3 & 147,0 & $87,2-621,9$ \\
\hline
\end{tabular}

Tab. 3. Cadmium-Konzentration im Nierenmark (nmol/g Trokkengewicht)

\begin{tabular}{|c|c|c|c|c|c|}
\hline & $\begin{array}{l}\text { Alter } \\
\overline{\mathbf{x}} \text { (a) }\end{array}$ & Median & $\overline{\mathbf{x}}$ & $\mathbf{s}$ & Bereich \\
\hline $\begin{array}{l}\text { Alle Proben } \\
n=68\end{array}$ & $63, \overline{7}$ & 186,4 & 238,6 & 200,1 & $34,7-913,7$ \\
\hline $\begin{array}{l}\text { Proben } \sigma \\
n=41\end{array}$ & 61,8 & 223,3 & 268,6 & 205,5 & $40,0-913,7$ \\
\hline $\begin{array}{l}\text { Proben }+ \\
n=27\end{array}$ & 64,6 & 174,4 & 193,1 & 186,0 & $34,7=898,6$ \\
\hline
\end{tabular}

Tab. 4. Gesamtkörperbelastung mit Cadmium ( $\mu \mathrm{mol}$ )

\begin{tabular}{llllll}
\hline & $\begin{array}{l}\text { Alter } \\
\overline{\mathbf{x}}(\mathrm{a})\end{array}$ & \multicolumn{2}{l}{ Median $\overline{\mathbf{x}}$} & $\mathrm{s}$ & Bereich \\
\hline $\begin{array}{l}\text { Alle Proben } \\
\mathrm{n}=68\end{array}$ & 63,7 & 51,6 & 60,8 & 45,6 & $13,2-303,9$ \\
$\begin{array}{l}\text { Proben } \delta \\
\mathbf{n}=41\end{array}$ & 61,8 & 65,4 & 76,6 & 51,2 & $14,9-303,9$ \\
$\begin{array}{l}\text { Proben } 9 \\
\mathbf{n}=27\end{array}$ & 64,6 & 32,7 & 36,8 & 17,9 & $13,2-77,2$ \\
\hline
\end{tabular}

Korrelationen zwischen der Cadmiumkonzentration in der Leber, in der Nierenrinde, im Nierenmark und der Gesamtkörperbelastung

Da im vorliegenden Untersuchungsgut die Cadmiumkonzentrationen in der Leber und in der Niere nicht normal verteilt auftraten, wurden die verteilungsfreien Rangkorrelationskoeffizienten nach Spearman berechnet. In Tabelle 5 sind die Korrelationskoeffizienten mit den dazugehörigen Signifikanzniveaus zusammengestellt.

EinfluB einer Leber- und/oder Nierenerkrankung auf die Cadmiumkonzentrationen in Leber, Nierenrinde, Nierenmark und die Körperbelastung

Die Diagnosen für die Erkrankungen wurden den Sektionprotokollen entnommen. Tabelle 6 zeigt die arithmetischen Mittelwerte der Cadmium-Gewebe-

Tab. 5. Korrelationen zwischen Alter (A), den Cadmiumkonzentrationen in der Leber (Cd-L), der Nierenrinde (CdNR), im Nierenmark (Cd-NM) und der CadmiumKörperbelastung (Cd-Last). Angegeben sind die Spearman'schen Rangkorrelationskoeffizienten und in Klammern die Signifikanzniveaus. $n=68$

\begin{tabular}{lccccc}
\hline & A & Cd-L & Cd-NR & Cd-NM & Cd-Last \\
\hline A & 1,0000 & & & & \\
Cd-L & 0,1555 & 1,0000 & & & \\
& $(0,2030)$ & & & & \\
Cd-NR & $-0,1500$ & 0,3665 & 1,0000 & & \\
& $(0,2196)$ & $(0,0027)$ & & & \\
Cd-NM & $-0,1495$ & 0,3474 & 0,6110 & 1,0000 & \\
& $(0,2212)$ & $(0,0045)$ & $(0,0000)$ & & \\
Cd-Last & $-0,1926$ & 0,5317 & 0,9002 & 0,5503 & 1,0000 \\
& $(0,1149)$ & $(0,0000)$ & $(0,0000)$ & $(0,0000)$ & \\
\hline
\end{tabular}

Tab. 6. Einfluß einer Lebererkrankung auf die Cadmium-Konzentrationen in der Leber (Cd-L), der Nierenrinde (CdNR), dem Nierenmark (Cd-NM) und auf die Körperlast (Cd-Last). Angegeben sind die arithmetischen Mittelwerte.

\begin{tabular}{|c|c|c|c|c|c|}
\hline \multirow[t]{2}{*}{ Lebererkrankung } & \multirow[t]{2}{*}{$\mathbf{n}$} & Cd-L & $\mathrm{Cd}-\mathrm{N}$ & Cd-NM & \multirow{2}{*}{$\begin{array}{l}\text { Cd-Las } \\
(\mu \mathrm{mol})\end{array}$} \\
\hline & & \multicolumn{3}{|c|}{ (nmol/g Trockengewicht) } & \\
\hline Keine & 12 & 23 & 516 & 178 & 79 \\
\hline Verfettung & 21 & 14 & 384 & 221 & 55 \\
\hline Stauungsleber & 15 & 24 & 392 & 254 & 55 \\
\hline Cirrhose & 9 & 19 & 370 & 329 & 54 \\
\hline Metastasen & 7 & 29 & 434 & 252 & 65 \\
\hline Ódem & 2 & 27 & 432 & 272 & 65 \\
\hline Einzelzellnekrose & 1 & 36 & 887 & 249 & 134 \\
\hline $\begin{array}{l}\text { Glykogenspeicher- } \\
\text { erkrankung }\end{array}$ & 1 & 16 & 310 & 140 & 40 \\
\hline Gesamt & 68 & 21 & 420 & 239 & 61 \\
\hline
\end{tabular}


Tab. 7. Einfluß einer Nierenerkrankung auf die CadmiumKonzentration in der Leber (Cd-L), der Nierenrinde (Cd-NR), im Nierenmark (Cd-NM) und auf die Körperlast (Cd-Last). Angegeben sind die arithmetischen Mittelwerte.

\begin{tabular}{lrrrrr}
\hline Nierenerkrankung & $\mathrm{n}$ & \multicolumn{2}{c}{ Cd-L } & Cd-NR Cd-NM & $\begin{array}{c}\text { Cd-Last } \\
\text { (nmol/g Trockengewicht) }\end{array}$ \\
\cline { 3 - 6 } & \multicolumn{5}{c}{$(\mu \mathrm{mol})$} \\
\hline Keine & 12 & 23 & 471 & 240 & 65 \\
Gefäßerkrankung & 30 & 19 & 407 & 236 & 58 \\
Schockniere & 6 & 31 & 491 & 424 & 75 \\
Diabetische & 6 & 19 & 345 & 214 & 47 \\
Glomerulosklerose & & & & & \\
Ödem & 5 & 12 & 270 & 164 & 40 \\
Metastasen & 2 & 9 & 211 & 168 & 28 \\
Glomerulonephritis & 1 & 25 & 109 & 224 & 26 \\
Verfettung & 1 & 49 & 179 & 50 & 48 \\
Nekrose & 1 & 6 & 87 & 68 & 14 \\
Nephrose unklarer & 1 & 12 & 803 & 528 & 109 \\
Genese & & & & & \\
Nephrocalcinose & 1 & 26 & 394 & 37 & 48 \\
Markfibrose & 1 & 36 & 2120 & 318 & 304 \\
Adenom & 1 & 17 & 232 & 69 & 77 \\
Gesamt & 68 & 21 & 420 & 239 & 61 \\
\hline
\end{tabular}

konzentration und der -Körperbelastung in Abhängigkeit von einer Lebererkrankung der Patienten. Tabelle 7 enthält die entsprechenden Werte in Abhängigkeit von einer Nierenerkrankung.

\section{Diskussion}

\section{Cadmium-Werte in der Leber und Niere}

Da die Proben an verschiedenen Stellen des Organs entnommen wurden, wird das Meßergebnis nicht nur durch die analytische Streubreite, sondern auch durch die biologische Varianz beeinflußt. Letztere fällt um so größer aus, je inhomogener der gesuchte Analyt im Gewebe verteilt ist. Wir fanden für die Leberproben einen durchschnittlichen intraindividuellen Variationskoeffizienten von $15,8 \%$, für die Nierenrindenproben von $25,6 \%$ und für die Nierenmarkproben von $22,3 \%$. Darin spiegelt sich die gleichmäßigere Verteilung des Cadmiums im relativ homogen strukturierten Lebergewebe im Vergleich zum Nierengewebe wider.

Die interindividuelle Streuung der Cadmium-Konzentrationen ist beträchtlich. In den von uns untersuchten Proben lag zwischen dem niedrigsten und höchsten gemessenen Wert in der Leber ein Faktor von 35, in der Nierenrinde von 24 und im Nierenmark von 26.

In unserem Untersuchungsgut zeigen Frauen gegenüber Männern im Durchschnitt niedrigere Cadmiumkonzentrationen, sowohl in der Leber als auch in der Nierenrinde und im Nierenmark. Drasch (12) konnte keinen Unterschied in den Cadmiumkonzentrationen in der Leber zwischen den Geschlechtern feststellen. Möglicherweise ist die Diskrepanz in einem unterschiedlichen Rauchverhalten in beiden Kollektiven zu suchen.

Die Cadmium-Konzentration im Nierenmark entspricht im Durchschnitt dem 0,56-fachen der Nierenrindenkonzentration. Bei einigen Gewebeproben fanden sich dagegen im Nierenmark höhere Werte als in der Nierenrinde. Dies deckt sich mit Beobachtungen von Drasch (13), der ebenfalls in einigen Fällen im Nierenmark höhere Werte als in der Nierenrinde ermittelt hat. Inwieweit eine solche Konstellation krankheitsbedingt ist, muß diskutiert werden (s. u.).

Vergleich der Meßdaten mit Literaturangaben und Problematik der Umrechnung der Ergebnisse von Feucht- auf Trockengewicht

Ein Vergleich unserer Meßergebnisse mit Literaturangaben ist schwierig, da fast alle Autoren ihre Werte auf das Feuchtgewicht beziehen. Wegen der Verdunstung von Wasser aus dem Gewebe haben wir es vorgezogen, unsere Proben zu lyophilisieren. Um trotzdem eine Ümrechnung zwischen den Ergebnissen vornehmen zu können, haben wir Faktoren für die Umrechnung von Feucht- auf Trockengewicht für Leber, Nierenrinde und Nierenmark ermittelt (s. Material und Methoden). Die von uns erhaltenen Faktoren stimmen mit den bei Friberg (1) angegebenen (Nierenrinde: ca. 0,20; Leber: ca. 0,28) gut überein.

Bei Kjellström (14) findet sich ein Vergleich der Cadmiumkonzentrationen in der Leber, die in drei verschiedenen Ländern gemessen wurden. Rechnet man die angegebenen Werte mit dem Faktor 0,24 auf Trokkengewicht und zusätzlich auf SI-Einheiten um, so erhält man 29,6 nmol/g für eine schwedische Population, $37,1 \mathrm{nmol} / \mathrm{g}$ für eine Bevölkerungsgruppe aus den USA und 111,3 nmol/g für eine japanische Population. Drasch (12) fand im Raum München, wiederum umgerechnet, $30,4 \mathrm{nmol} / \mathrm{g}$. In den hier untersuchten Proben fand sich ein Median von $16,0 \mathrm{nmol} / \mathrm{g}$ Trockengewicht.

Literaturangaben über die Cadmium-Konzentrationen in der Niere beziehen sich meist nur auf die Nierenrinde. Das Verhältnis zwischen der Cadmiumkonzentration in der Nierenrinde zur Konzentration im Nierenmark wird mit 2:1 angegeben (1). Wir fanden in unseren Proben ein durchschnittliches Verhältnis von 1,8:1. Für die Cadmiumkonzzentration in der Nierenrinde findet sich bei Elinder (1) eine Literaturzusammenstellung, welche Studien aus 10 Ländern berücksichtigt. Die Cadmitümkonzentrationen 
liegen demnach im Mittel zwischen 889 und 1779 $\mathrm{nmol} / \mathrm{g}$ Trockengewicht, lediglich in Japan fanden sich im Mittel $4448 \mathrm{nmol} / \mathrm{g}$ Trockengewicht. Scott (16) ermittelte in Großbritannien $683 \mathrm{nmol} / \mathrm{g}$ Trockengewicht, Drasch (12) gibt für den Raum München als Median $974 \mathrm{nmol} / \mathrm{g}$ Trockengewicht an. Schiele fand im bayrischen Raum als arithmetisches Mittel 439 nmol/g Trockengewicht (7). Alle oben genannten Werte wurden von uns auf SI-Einheiten und Trockengewicht umgerechnet. Bei den hier vorliegenden Proben aus dem Raum Augsburg fand sich als Median in der Nierenrinde $315 \mathrm{nmol} / \mathrm{g}$ Trockengewicht.

Um die Problematik der Umrechnung von Feuchtauf Trockengewicht näher zu beleuchten, wurde die Verdunstung von Wasser aus Nierenrindenproben näher untersucht. Es wurde ein $1 \mathrm{mg}$ schweres Stück feuchte Nierenrinde (dies entspricht etwa der Menge, die bei einer direkten Vermessung im AAS-Gerät eingesetzt wird) in eine geschlossene Analysenwaage gelegt. Diese Probe verlor in nur 3 Minuten $25 \%$ ihres Gewichtes. Bei einem 16.2 mg schweren Stück feuchter Nierenrinde fanden wir innerhalb von 5 Minuten eine Abnahme des Gewichtes um 5\%. Eine Umrechnung von Feucht- auf Trockengewicht erscheint deshalb problematisch und die von uns im Vergleich zur Literatur gefundenen niedrigeren Werte dürften sich zumindest teilweise aus diesem Umstand erklären. Gegen die Vermessung von feuchten Gewebeproben spricht auch die unterschiedliche Hydratation der Organe bei verschiedenen Krankheitszuständen. So liegt der Wassergehalt z. B. bei Stauungsleber oder Nierenödem naturgemä $B$ höhier, während er andererseits bei Lebercirrhose oder Schrumpfniere niedriger liegt als in gesunden Organen. Ebenso ist zu beachten, daß es im Finalstadium zu einer Überwässerung der Niere kommen kann. Aus diesen grundsätzlichen Überlegungen sollte unseres Erachtens der Messung lyophilisierter Proben der Vorzug gegeben werden.

Bei Literaturvergleichen muß zudem berücksichtigt werden, ob die Werte als arithmetisches Mittel oder als Median ausgedrückt sind. Der Median liegt, da es sich im allgemeinen um log=normale Häufigkeitsverteilungen handelt, niedriger als das arithmetische Mittel. In unserer Untersuchung betrugen die Mediane bei den Leberproben $77,6 \%$, bei den Nierenrinden$75,1 \%$ und bei den Nierenmarkproben $78,1 \%$ des jeweiligen arithmetischen Mittels. Elinder (1) gibt das Verhältnis von geometrischem $\mathrm{zu}$ arithmetischem Mittel für die Leber mit $80 \%$ und für die Niere mit $85 \%$ an.

In der Literatur angegebene Werte für die Gesamtkörperbelastung liegen in Europa und den USA zwischen 44,5 und 266,8 $\mu \mathrm{mol}$. Für Japan wurden z.T. wesentlich höhere Werte um $711 \mu \mathrm{mol}$ angegeben (9). Drasch errechnete einen Wert von $137 \mu \mathrm{mol}$. Aus den hier gewonnenen Daten wurde ein Cadmiumbelastung von durchschnittlich $60,8 \mu \mathrm{mol}$ errechnet. Dieser Wert liegt im unteren Bereich der Literaturangaben.

Korrelationen zwischen der Cadmiumkonzentration in der Leber, in der Nierenrinde, im Nierenmark und der Gesamtkörperbelastung

Zwischen der Cadmium-Konzentration der Leber und den Cadmium-Konzentrationen in der Nierenrinde und dem Nierenmark bestehen etwa gleichwertige schwache, jedoch signifikante Korrelationen (Tab. 5). Eine stärkere Korrelation findet sich zwischen den Cadmium-Konzentrationen in der Nierenrinde und dem Nierenmark $\left(r_{s}=0,61\right)$. Obwohl die CadmiumKörperlast gleichermaßen aus den Konzentrationen in der Leber und der Nierenrinde berechnet wurde, korreliert sie am besten mit der Nierenrindenkonzentration $\left(\mathrm{r}_{\mathrm{s}}=0,9\right)$, da die Cadmiumkonzentration der Nierenrinde etwa 20-mal so hoch wie die der Leber ist. Eine Korrelation der Gewebe-Cadmiumkonzentration mit dem Alter ist nicht gegeben, da es sich bei dieser Untersuchung hauptsächlich um ältere Patienten handelte (Durchschnittsalter 63,7 Jahre). Die negative Korrelation zwischen Alter und Nierenwerten spiegelt die Abnahme der Nieren-Cadmiumkonzentration ab dem 6 . Lebensjahrzehnt wider (1).

Einfluß einer Leber- und Nierenerkrankung auf die Cadmium-Konzentrationen in der Leber und der Niere

Eine Beurteilung des Einflusses einer Leber- und/oder Nierenerkrankung auf die Cadmiumkonzentrationen in der Leber und der Niere muß mit großer Vorsicht erfolgen. Zum einen ist $\mathrm{zu}$ berücksichtigen, daß Raucher über den Tabakrauch Cadmium aufnehmen und somit höhere Cadmiumkonzentrationen im Gewebe aufweisen. Elinder (1) ermittelte nach Auswertung von Studien aus sechs Ländern eine durchschnittliche Erhöhung der Cadmium-Nierenrindenkonzentration bei Rauchern um ca. ein Drittel. In der Leber erhöhte sich die Cadmiumkonzentration bei Rauchern einer schwedischen Population um durchschnittlich zwei Drittel (15). Damit kann der Einfluß einer Leber- oder Nierenerkrankung überlagert werden. Leider war es uns nicht möglich, eine nachträgliche Raucheranamnese durchzuführen. Zum anderen spielt auch das Lebensalter eine Rolle, da die Cadmium-Nierenrindenkonzentration bis zum Alter von ca. 50 Jahren ansteigt, um dann wieder abzufallen (1). 
Dieser Abfall könnte jedoch selbst krankheitsbedingt sein. Unter diesen Vorbehalten soll eine Interpretation der Ergebnisse versucht werden.

Aus Tabelle 6 lassen sich folgende Schlüsse ziehen: Bei Personen ohne Lebererkrankung finden sich relativ hohe Konzentrationen in der Nierenrinde, bei relativ niedrigen Konzentrationen im Nierenmark. Möglicherweise ist in diesen Fällen die ausreichende Bildung des Cadmium-bindenden Thioneins $\left(M_{\mathrm{r}}\right.$ ca. 6500 ) in der Leber verantwortlich für die Deponierung und Fixierung des Cadmiums in der Nierenrinde. Insgesamt betrachtet scheint bei Patienten mit einer Lebererkrankung die Gesamtkörperbelastung niedriger zu liegen als bei Patienten ohne Leberschaden. Eine Ausnahme bildet der Fall einer Einzelzellnekrose mit deutlich über dem Durchschnitt liegenden Werten für die Nierenrinde und die Körperlast. In allen übrigen Fällen findet sich eine verminderte Körperbelastung mit Cadmium. Diese resultiert (wie aus Tabelle 6 ersichtlich) prịmär aus einer Verminderung der Cadmium-Konzentration in der Nierenrinde. Eine reduzierte Cadmiumkonzentration in der Leber findet sich bei einem Fall mit Glykogenspeichererkrankung, außerdem bei Verfettung der Leber sowie in geringem Ausmaß bei Lebercirrhose. Bei den Patienten mit Lebercirrhose fällt die fast gleichmäßige Verteilung des Cadmiums zwischen Nierenrinde und Nierenmark auf. Dies gilt jedoch nur bei Betrachtung der Durchschnittswerte; prüft man die Fälle einzeln, so trifft diese Konstellation bei etwa der Hälfte der Cirrhosepatienten zu, in drei Fällen war die Konzentration im Nierenmark höher als die in der Nierenrinde. Offensichtlich erfolgt die Deponierung des Cadmiums in der Niere bei Thioneinmangel in anderer Weise als bei ausreichender Thionein-Bildung der Leber, da das nicht an Thionein gebundene Cadmium beweglicher ist.

Betrachtet man in Tabelle 7 den Einfluß einer Nierenerkrankung auf die Cadmiumkonzentration in der Leber, so fallen zwei niedrige Werte bei Patienten mit Metastasen und Nierennekrose auf. Bei einem Patienten mit Metastasen in den Nieren war auch die Leber mitbeteiligt, der Patient mit Nierennekrose zeigte eine beginnende Lebercirrhose. Damit wäre zumindest im letzteren Fall die niedrige Cadmiumkonzentration in der Leber zu erklären. Weiterhin finden sich relativ niedrige Cadmium-Leberwerte bei Patienten mit Nierenödem und bei einem Patienten mit Nephrose. Höhere Leberwerte, als dem Durchschnitt der Patienten ohne Nierenerkrankung entspricht, weisen je ein $\mathrm{Pa}$ tient mit Nierenverfettung, mit Markfibrose und mit Schockniere auf.

Betrachtet man die Auswirkungen einer Nierenerkrankung auf die Cadmium-Werte in der Niere, so fallen die niedrigen Werte bei Glomerulonephritis, Nierenverfettung und Nekrose auf. Höhere Cadmium-Werte in der Niere, als sie durchschnittlich bei Patienten ohne Nierenerkrankung vorliegen, wurde bei einem Nephrosepatienten und besonders bei einem Patienten mit Markfibrose festgestellt. Die Patienten mit Schockniere weisen bei der Bildung des Mittelwertes eine relativ gleichmäßige Verteilung des Cadmiums zwischen Nierenrinde und Nierenmark auf. Drei dieser Patienten hatten eine höhere Konzentration im Nierenmark als in der Nierenrinde. Als Ursache kommt die bei Schockniere auftretende Minderperfusion mit daraus resultierender Tubulusnekrose in Betracht.

Die niedrigsten Werte für die Gesamtkörperbelastung fanden sich bei einem Patienten mit Glomerulonephritis, bei einem Patienten mit Nierennekrose und bei den Patienten mit Nierenmetastasen. Ein deutlich erhöhter Wert für die Gesamtkörperbelastung zeigte ein Patient mit Markfibrose.

Zusammenfassend läßt sich sagen, daß eine Ümrechnung der Cadmium-Konzentrationen von Feucht- auf Trockengewicht aus den oben genannten Gründen praktisch nicht möglich ist. Trotzdem zeigen unsere Ergebnisse, daß die Cadmiumbelastung im Raum Augsburg eher niedrig liegt und im Vergleich mit Literaturangaben im unteren angegebenen Bereich angesiedelt ist.

\section{Danksagung}

Wir danken den Mitarbeitern des Pathologischen Instituts im Hause für die zuverlässigen Probennahmen. Insbesondere gilt unser Dank dem Chefarzt des Pathologischen Institutes Herrn Prof. Dr. R. Backmann für die wohlwollende Unterstützung dieser Arbeit. 


\section{Literatur}

1. Friberg, L. Elinder, C. A., Kjellström, T. \& Nordberg, G. F. (Hrsg.) (1984) Cadmium and Health. A Toxicological and Epidemiological Appraisal CRC Press, Inc. Boca Raton, Florida.

2. Umweltbundesamt, Berlin (1980) Cadmiumbericht.

3. Takenaka, S., Oldiges, H., Hochreiner, D. \& Oberdörster, G. J. (1983) Nat. Cancer Inst. 70, 367-373.

4. Friberg, L. (1950) Acta Med. Scand. 138, Suppl. 240, 1-124.

5. Lauwerys, R. R., Buchet, J. P., Roels, H. A., Browers, J. \& Stanescu, P. (1974) Arch. Environ. Health 28, 145-148.

6. Gruhl, H. \& Gilek, U. (1987) Ärztl. Lab. 33, 186-190.

7. Schiele, R., Schaller, H. K. \& Valentin, H. (1983) Nierenund Hochdruckkrankheiten 12, 242-249.

8. Friberg, L., Piscator, M., Nordberg, G. F. \& Kjellström, T. (1974) Cadmium in the Environment. 2nd edition CRC Press Cleveland.

9. Kjellström, T. \& Nordberg, G. F. (1978) Environ. Res. 16, $248-269$.

10. Lauwereys, R. (Hrsg.) (1982) The Toxicology of Cadmium. Commission of the European Communities Luxemburg.

11. Swartengren, M., Elinder, C. G., Lind, B. \& Friberg, L. In: 1. c. (1) Vol. 1, S. 91.

12. Drasch, G. (1983) Habilitationsschrift Ludwig-Maximilians Universität München.

13. Drasch, G. (1987) Persönliche Mitteilung.

14. Kjellström, T. (1979) Environ Health Perspect. 28, 169197.

15. Elinder, C. G., Kjellström, T., Friberg, L., Lind, B. \& Linnman, L. (1976) Arch. Environ. Health 31, 292-302.

16. Scott, R., Aughey, E., Fell, G. S. \& Quinn, M. J. (1987) Human Toxicol. 6, 111-119.

Dr. Hartmut Gruhl

Institut für Laboratoriumsmedizin

Zentralklinikum

Stenglinstraße 2

D-8900 Augsburg 


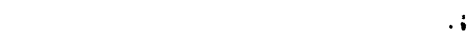

